\title{
Approximate Analytical Models of Shock-Wave Structure at Steady Mach Reflection
}

\author{
Mikhail V. Chernyshov (D), Karina E. Savelova * and Anna S. Kapralova \\ Department of Plasma and Gas Dynamics, Baltic State Technical University "VOENMEH", 1 Pervaya \\ Krasnoarmeyskaya Ul., 190005 Saint Petersburg, Russia; chernyshov_mv@voenmeh.ru (M.V.C.); \\ kapralovaa@yahoo.com (A.S.K.) \\ * Correspondence: karinkamurz@yandex.ru; Tel.: +7-9218878419
}

check for updates

Citation: Chernyshov, M.V.;

Savelova, K.E.; Kapralova, A.S.

Approximate Analytical Models of Shock-Wave Structure at Steady Mach Reflection. Fluids 2021, 6, 305

https://doi.org/10.3390/

fluids6090305

Academic Editor: Pier Marzocca

Received: 12 July 2021

Accepted: 25 August 2021

Published: 28 August 2021

Publisher's Note: MDPI stays neutral with regard to jurisdictional claims in published maps and institutional affiliations.

Copyright: (c) 2021 by the authors. Licensee MDPI, Basel, Switzerland. This article is an open access article distributed under the terms and conditions of the Creative Commons Attribution (CC BY) license (https:// creativecommons.org/licenses/by/ $4.0 /$ )
Abstract: In this study, we obtain the comparative analysis of methods of quick approximate analytical prediction of Mach shock height in planar steady supersonic flows (for example, in supersonic jet flow and in narrowing channel between two wedges), that are developed since the 1980s and being actively modernized now. A new analytical model based on flow averaging downstream curved Mach shock is proposed, which seems more accurate than preceding models, comparing with numerical and experimental data.

Keywords: shock reflection; supersonic flow; Mach stem

\section{Introduction}

Since two types of shock reflection (the regular reflection, and the irregular one, commonly called Mach reflection, were discovered both in unsteady [1] and steady [2] shock/surface, shock/symmetry line and symmetric shock/shock interactions, a large number of fruitful studies was obtained (see, for example, reviews of the current state-ofthe-art in different periods discussed in [3-6]). The great growth of interest in shock/surface reflections occurred in the 1940s due to research of damaging mechanical action of largescale charges of high explosives and nuclear weapons. Great physicists and mathematicians [7-10], such as J. von Neumann, R. Courant, K.O. Friedrichs, L.D. Landau, and Ya.B. Zel'dovich, were involved in that studies of vast civil and military importance, and they achieved sufficient progress in our knowledge. Two basic criteria of regular/Mach shock reflection transition (the "von Neumann criterion", which corresponds to the formation of so-called stationary Mach configuration with normal Mach shock, and "detachment criterion", which corresponds to maximum flow reflection angle at regularly reflected shock) were established [7]; with some additions and remarks, we apply them to flow analysis until this time. Some valuable results were obtained later: the impossibility of regular reflection in inviscid steady axisymmetric flow was proven [11]; hysteresis phenomenon in regular/Mach reflection transition was shown experimentally [12,13] and numerically [14-16]. According to the modern point of view [6,17], change of reflection type in unsteady flow usually corresponds to the "detachment criterion"; steady reflection transition usually corresponds to von Neumann one.

Not only the type of shock reflection but also geometric features of the forming shockwave structure, including its key parameter (Mach stem height or Mach shock size), are of vast importance nowadays. Geometrical parameters of occurring shocks and other gasodynamic discontinuities define the aggregate (integral) features downstream, as well as flow features in its various regions. So, a steady Mach reflection in supersonic gas jet flows, convergent supersonic inlets and other aerospace facilities leads to the formation of complex shock-wave structure that divides the supersonic stream into two regions with very different values of stagnation pressure, dynamic pressure, flow velocity, static temperature, gas density, acoustic impedance, and many other flow characteristics of 
great engineering importance [18]. Such parameters as stagnation pressures and gas static temperatures across the slipstream that emanates from the triple point can differ in dozens of times [18,19]. The Mach stem size is traditionally considered the key parameter that helps to estimate volumes of both above-mentioned regions, to compare their influence, and to analyze the shock-wave structure and flow features of the downstream Mach reflection point ("triple point") as a whole. However, the estimation of that important flow structure parameter should be very accurate because different widespread CFD software also gives us very different, even contradictory, results (see, for instance, the discussion on the ambiguity of the solution and the performability of the so-called "negative" triple configurations in [20-23]). It concerns both "commercial" and "homemade" ("researcher's") hydrocodes because their solutions are very sensitive to initial and boundary conditions, the type of solver, the accepted model of turbulence, and so on. Despite this, simple and easy-to-use analytical models of shock-wave structures are needed for fast and reliable estimation and analysis of flow features as a whole.

A new splash of interest in the analytical prediction of the Mach stem size appears now again [24-28] because of progress in the design of aviation and rocket engines (including detonation ones [29]) and also in high supersonic and hypersonic flights. As it was remarked and patented in [30], treated analytically in [31] and numerically in [32], it can be used in design of the prospective combined ramjet engine. The high temperature of the flow immediately after the Mach stem can initiate combustion or detonation of fuel mixture, so the cycle of the ramjet detonation engine is most prospective for it. On the contrary, relatively large stagnation pressure after the reflected shock makes that part of flow most efficient in the traditional ramjet engine. Therefore, it is crucially important to know the Mach stem height and the shape of the slipstream that separates two flow regions that are to be used in different ways. The most evident way to separate those flow regions in practice seems to be situating a thin rigid wall between them, which repeats the shape of the emanating slipstream. However, to realize it, it is necessary to know the Mach stem height and the shape of the emanating slipstream. Some important problems of supersonic jet functionality distance, the design of supersonic inlets, and so on can also be reduced by Mach stem height determination.

The proposed model does not take into account the real gas effects, impulse energy efflux due to combustion and detonation initialized by high temperature growth behind the Mach stem [18,19], non-equilibrium flows [33], or, for example, plasma formation [34]. An impulse energy influx can be included into this mathematical model applying the Zel'dovich-Döring-von Neumann (ZDN) detonation model and corresponding relations for the Mach shock wave with the energy efflux $[35,36]$. As preliminary results show, heat efflux leads to the formation of a Mach reflection in situations where only regular reflection is theoretically possible in flows without chemical reactions. Heat efflux also leads to a sufficient increase of the Mach stem size. Real gas effects that result in a decrease in the "effective" gas adiabatic index at Mach stem shifts the regular/Mach transition border at the same direction and also increases the Mach stem height but not sufficiently. Ionization and plasma formation in flows with Mach reflection can be subjects of further studies.

In this study, we conduct a comparative analysis of methods of quick approximate analytical prediction of the Mach shock height in the planar steady supersonic flow and both the axisymmetric and asymmetric one, which have been developed since the 1980s and are still being actively modernized now. A new analytical model based on modern results in the shock interaction theory is proposed, and its results are compared with preceding models, numerical data, and experimental data. Potential results of the proposed models can be applied to supersonic gas jet flow studies, as well as for designing convergent supersonic inlets, supersonic nozzles, prospective ramjets, and other aerospace facilities. It can be useful also for verifying CFD hydrocodes and further progress in numerical methods (for example, it is applicable to the adaptation of the classic Godunov method for steady supersonic flows, which belongs to Ivanov and Kraiko, see [37,38]). 


\section{Model and Methods}

\subsection{Triple Point Problem}

Let us consider the single Mach reflection from a symmetry plane in inviscid steady supersonic flow of perfect gas, which takes place in the narrowing channel between two wedges (Figure 1a) or in an overexpanded planar jet flow out of a supersonic nozzle (Figure 1b).

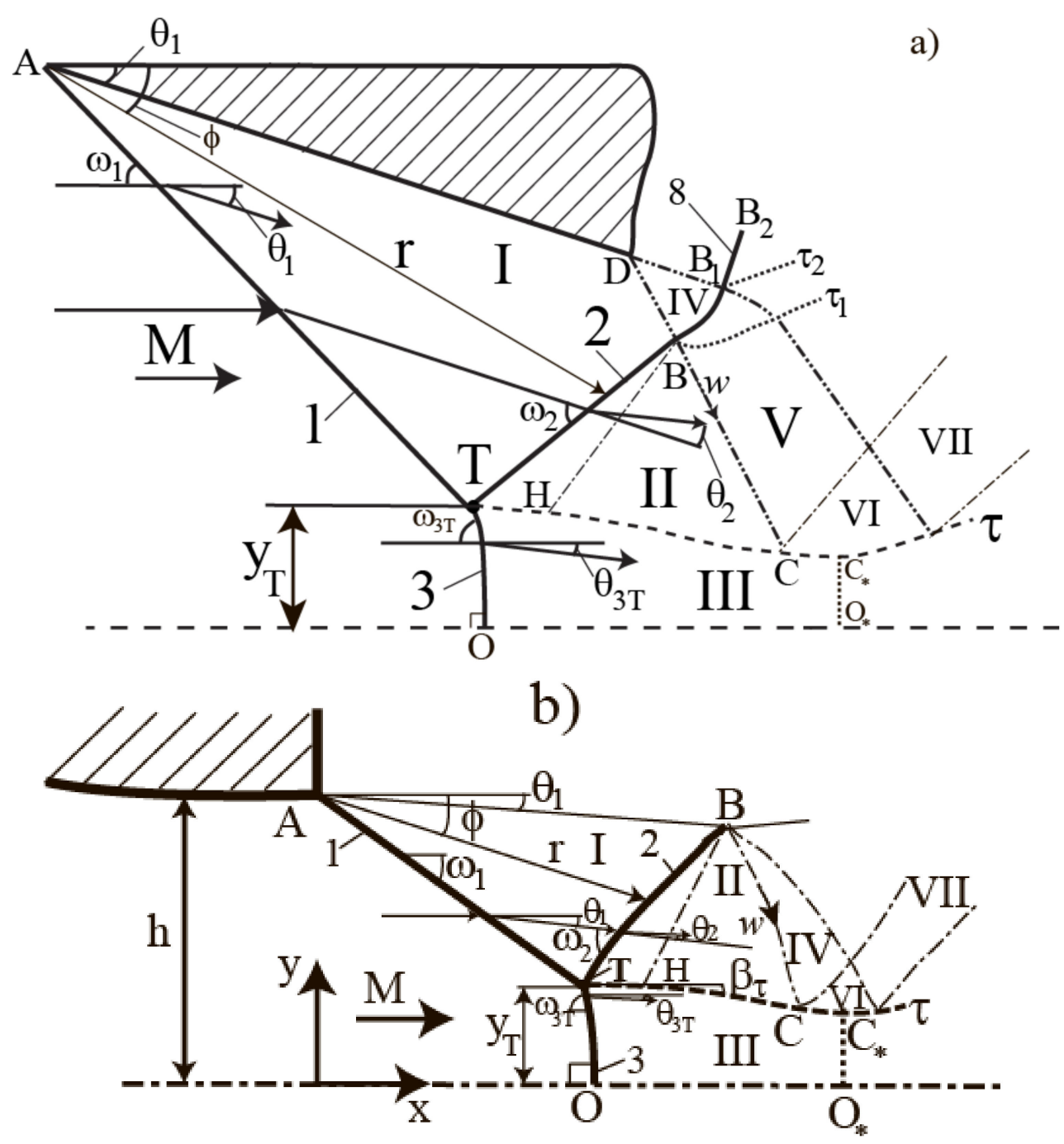

Figure 1. Patterns of flows with Mach reflection: (a) in a narrowing channel between two wedges; (b) in overexpanded gas jet flow. Here, $M$ is the Mach number of unperturbed flow; $1(A T)$ is the incident shock; 2 (TB) is the reflected one; 3 (TO) is the main shock (Mach stem); $T$ is the triple point; $\tau$ is the emanating slipstream; $\beta_{\tau}$ is the local slope angle of the slipstream $\tau ; \theta_{1}$ and $\theta_{2}$ are flow deflection angles on shocks 1 and $2 ; \theta_{3 T}$ is the flow deflection angle on shock 3 at triple point $T ; \omega_{1}$ and $\omega_{2}$ are slope angles of the shocks 1 and 2 to flow velocity vectors upstream them; $\omega_{3 T}$ is the slope angle of the shock 3 in triple point $T ; h$ is the width of the channel; $y_{T}$ is the sought Mach stem height; $(r, \phi)$ are the distance and the polar angle in cylindrical coordinate system centered in point $A ; H B$ is the first-family acoustical characteristic that comes into point $B$; I if flow region behind the incident shock 1 ; II (BTC) is the flow region downstream the reflected shock that can be approximated as Prandtl-Meyer flow with straight first-family characteristics; III is the flow region behind the Mach stem ("virtual nozzle" with critical section $C_{*} O_{*}$ ); IV is the expansion fan; V (Figure 1a) is the refracted expansion fan resulting in the interaction of wave IV with the curvilinear shock $B B_{1}$; VI is the reflection zone o expansion wave; VII is the reflected wave; $B C$ is the first acoustical characteristic of the expansion wave that turns the slipstream $\tau ; \tau_{1}$ and $\tau_{2}$ (Figure 1 a) are weak tangential discontinuities; $8\left(B_{1} B_{2}\right.$, Figure 1a) is the resulting shock after the interaction with the expansion wave IV. 
The incident shock $1(A T)$ forms in the unperturbed flow with Mach number $M$. The incident shock strength $J_{1}$ (relation of the static pressures downstream the shock and before it) and flow deflection angle $\theta_{1}$ are the basic characteristics of the shock 1 ; they correlate between themselves as it follows:

$$
\tan \left|\theta_{1}\right|=\sqrt{\frac{(1+\varepsilon) M^{2}-J_{1}-\varepsilon}{J_{1}+\varepsilon}} \frac{(1-\varepsilon)\left(J_{1}-1\right)}{(1+\varepsilon) M^{2}-(1-\varepsilon)\left(J_{1}-1\right)}
$$

Here, $\varepsilon=(\gamma-1) /(\gamma+1)$, and $\gamma$ is the ratio of gas specific heats $(\gamma=1.4$ in all further calculations).

The reflection of the shock 1 is Mach one according to the von Neumann criterion. It means that the incident shock strength belongs to the range

$$
J_{N} \leq J_{1} \leq J_{T}
$$

The minimum shock strength $J_{N}$ corresponds to the formation of the so-called "stationary Mach configuration" with normal Mach stem TO. Equation (3) [39]

$$
\begin{gathered}
\sum_{n=0}^{3} E_{n} J_{N}^{n}=0 \\
E_{3}=1-\varepsilon, E_{2}=-\left[\left(1+\varepsilon-\varepsilon^{2}+\varepsilon^{3}\right) M^{2}+(1-\varepsilon)\left(1-\varepsilon+\varepsilon^{2}\right)\right] \\
E_{1}=\varepsilon\left[(1+\varepsilon) M^{2}+1-\varepsilon\right] \cdot\left[(1-\varepsilon) M^{2}-2+\varepsilon\right] \\
E_{0}=(1-\varepsilon)\left(M^{2}-1\right)\left((1+\varepsilon) M^{2}-\varepsilon\right)
\end{gathered}
$$

determines the incident shock strength value that corresponds to the Mach/regular reflection transition. The largest admissible shock strength $J_{1}=J_{T}$ corresponds to triple-shock configuration with reflected shock $2(T B)$, which is normal to the flow upstream of it. This transitional shock strength value satisfies the equation $[18,19]$

$$
M^{4}-r M^{2}+\left(J_{T}-1\right)\left(J_{T}+2-\varepsilon\right) /(1-\varepsilon)=0,
$$

$$
r=\left(J_{T}-1\right)\left(J_{T}+2-\varepsilon\right) /\left(J_{T}+\varepsilon\right)+\left(J_{T}+\varepsilon\right) /(1+\varepsilon)+\left(1+\varepsilon J_{T}\right)^{2} /\left[(1-\varepsilon)\left(J_{T}+\varepsilon\right)^{2}\right]
$$

Shock reflections at $J_{1}>J_{T}$ are known as the von Neumann one and the Vasilev one [6]; usually, they do not appear in steady flows, especially at moderate and large Mach numbers.

On the stipulation that the inequality in Equation (2) is satisfied, a single Mach reflection appears in the triple point $T$. The condition of flow collinearity and the equality of static pressures across the emanated slipstream $\tau$ lead to the following system of equations that determines the parameters of the reflected shock $2(T B)$ and the main shock (Mach stem) $3(T O)$ :

$$
\theta_{1}+\theta_{2 T}=\theta_{3 T}, J_{1} J_{2 T}=J_{3 T}
$$

Here, $J_{2 T}$ and $J_{3 T}$ are, correspondingly, the strengths of shocks 2 and 3 in point $T$; the following relations analogous to Equation (1) determine the flow deflection angles $\theta_{2 T}$ and $\theta_{3 T}$ in that point:

$$
\begin{aligned}
\tan \left|\theta_{2 T}\right| & =\sqrt{\frac{(1+\varepsilon) M_{1}^{2}-J_{2 T}-\varepsilon}{J_{2 T}+\varepsilon}} \frac{(1-\varepsilon)\left(J_{2 T}-1\right)}{(1+\varepsilon) M_{1}^{2}-(1-\varepsilon)\left(J_{2 T}-1\right)}, \\
\tan \left|\theta_{3 T}\right| & =\sqrt{\frac{(1+\varepsilon) M^{2}-J_{3 T}-\varepsilon}{J_{3 T}+\varepsilon}} \frac{(1-\varepsilon)\left(J_{3 T}-1\right)}{(1+\varepsilon) M^{2}-(1-\varepsilon)\left(J_{3 T}-1\right)} .
\end{aligned}
$$

(deflection angles are positive if flow turns counterclockwise). Flow Mach numbers $\left(M_{1}\right.$ after the incident shock 1 , as well as $M_{2 T}$ and $M_{3 T}$ after the corresponding shocks in the triple point vicinity), satisfy the following formulas: 


$$
\begin{gathered}
M_{1}=\sqrt{\frac{\left(J_{1}+\varepsilon\right) M^{2}-(1-\varepsilon)\left(J_{1}^{2}-1\right)}{J_{1}\left(1+\varepsilon J_{1}\right)}}, M_{2 T}=\sqrt{\frac{\left(J_{2 T}+\varepsilon\right) M_{1}^{2}-(1-\varepsilon)\left(J_{2 T}^{2}-1\right)}{J_{2 T}\left(1+\varepsilon J_{2 T}\right)}}, \\
M_{3 T}=\sqrt{\frac{\left(J_{3 T}+\varepsilon\right) M^{2}-(1-\varepsilon)\left(J_{3 T}^{2}-1\right)}{J_{3 T}\left(1+\varepsilon J_{3 T}\right)}} .
\end{gathered}
$$

In further considerations, we postulate that the flow behind the incident shock 2 should be supersonic (i.e., $M_{2} \geq 1$ ). It leads to following limitation of incident shock strength:

$$
J_{N} \leq J_{1} \leq J_{S}
$$

which is a little stricter than Equation (2). The incident shock strength $J_{S}$ corresponds to the critical flow velocity behind the irregularly reflected shock. The high-order algebraic equation for its value is more complicated. (It is of the 10th order in regard to $M^{2}$ and of the 14 th order in regard to $J_{S}$. See, for example, its numerical solution and analysis in $[18,23]$.)

The angles $\omega_{1}, \omega_{2 T}$, and $\omega_{3 T}$ of shock inclination to flow direction upstream them (see Figure 1a,b) can be calculated as it follows:

$J_{1}=(1+\varepsilon) M^{2} \sin ^{2} \omega_{1}-\varepsilon, J_{2 T}=(1+\varepsilon) M_{1}^{2} \sin ^{2} \omega_{2 T}-\varepsilon, J_{3 T}=(1+\varepsilon) M^{2} \sin ^{2} \omega_{3 T}-\varepsilon$

As the reflected shock 2 and the Mach stem 3 have non-zero geometrical curvature, their strengths $J_{2}$ and $J_{3}$ are variable functions that change from the values $J_{2 T}$ and $J_{3 T}$ in the triple point to the values $J_{2 B}$ and $J_{3 O}$ in points $B$ and $O$, correspondingly. The flow deflection angles $\theta_{2}$ and $\theta_{3}$ in voluntary points of shock fronts obey as it follows:

$$
\begin{aligned}
\tan \theta_{2} & =\sqrt{\frac{(1+\varepsilon) M_{1}^{2}-J_{2}-\varepsilon}{J_{2}+\varepsilon}} \frac{(1-\varepsilon)\left(J_{2}-1\right)}{(1+\varepsilon) M_{1}^{2}-(1-\varepsilon)\left(J_{2}-1\right)}, \\
\tan \left|\theta_{3}\right| & =\sqrt{\frac{(1+\varepsilon) M^{2}-J_{3}-\varepsilon}{J_{3}+\varepsilon}} \frac{(1-\varepsilon)\left(J_{3}-1\right)}{(1+\varepsilon) M^{2}-(1-\varepsilon)\left(J_{3}-1\right)},
\end{aligned}
$$

as well as shock slope angles $\omega_{2}$ and $\omega_{3}$ in their voluntary points:

$$
J_{2}=(1+\varepsilon) M_{1}^{2} \sin ^{2} \omega_{2}-\varepsilon, J_{3}=(1+\varepsilon) M^{2} \sin ^{2} \omega_{3}-\varepsilon,
$$

and Mach numbers $M_{2 T}$ and $M_{3 T}$ in regions II and III just after the curved shocks:

$$
M_{2}=\sqrt{\frac{\left(J_{2}+\varepsilon\right) M_{1}^{2}-(1-\varepsilon)\left(J_{2}^{2}-1\right)}{J_{2}\left(1+\varepsilon J_{2}\right)}}, M_{3}=\sqrt{\frac{\left(J_{3}+\varepsilon\right) M^{2}-(1-\varepsilon)\left(J_{3}^{2}-1\right)}{J_{3}\left(1+\varepsilon J_{3}\right)}} .
$$

Therefore, Equations (1) and (4)-(7) at additional restrictions of Equation (2) or Equation (8) allow us to calculate the parameters of all shocks and flow features behind them. The decision at the triple point is evident and exact for the inviscid flow. It is wellstudied parametrically [18,40-42] and often used in various analytical models [24-28,43-45]. However, it does still not determine the geometrical size of the Mach stem and the shape of other shocks and discontinuities consequently. This problem remains unsolved [46] and needs consideration of other details of the complex flow.

\section{2. "Virtual Nozzle" below the Slipstream}

The slipstream $\tau$ (also known as the contact discontinuity or the tangential one), which is directed at angle $\beta_{3 T}=\theta_{1}+\theta_{2 T}$ to the horizontal plane in point $T$, bounds the region III of initially subsonic flow. According to von Neumann criterion of Mach reflection, $\beta_{3 T}<0$. The Guderley reflection that theoretically appears at $\beta_{3 T}>0$, in fact, does not form in real steady flows [6].

Due to small flow gradients across region III, this flow can be usually treated as isentropic and quasi-one-dimensional one, analogous to the quasi-one-dimensional flow along 
convergent-divergent ("de Laval") supersonic nozzle. The analogy with the supersonic nozzle is even more complete because the slipstream $\tau$ turns upside down under the influence of the expansion fan IV (Figure 1b) or the refracted expansion fan V (Figure 1a) so that its slope angle $\beta_{\tau}=0$ at point $C_{*}$. Contemporarily, as laboratory $[12,13]$ and numerical $[47,48]$ tests witness, the flow velocity in the subsonic pocket $T O O_{*} C_{*}$ after the section $O_{*} C_{*}$ becomes supersonic. Thus, the term "virtual nozzle" [48] is the relevant characteristic of the flow in region III.

The coincidence of the contact discontinuity turn, and the transition of flow downstream the Mach stem to supersonic speeds is the key condition in numerous analytical [24-28,43-46] and numerical [47-49] models for the estimation of Mach stem size $y_{T}$. As the Mach stem estimation is too small, the gas stream in zone III reaches a critical velocity sooner than the turn of the slipstream occurs; on the contrary, if current estimation of $y_{T}$ is too large, the slipstream turns at a subsonic flow below it $\left(\beta_{\tau}=0\right.$ at $\left.M_{3}<1\right)$.

As we accept the quasi-one-dimensional model for region III after the Mach shock, the width $y$ of this region determines the Mach number $M_{3 \tau}$ below the slipstream in the corresponding section:

$$
y_{*} / y=q\left(M_{3 \tau}\right)
$$

so that

$$
y / y_{T}=q\left(M_{3}\right) / q\left(M_{3 \tau}\right) .
$$

Here, $q(M)=M \cdot\left[1+\varepsilon\left(M^{2}-1\right)\right]^{-1 / 2 \varepsilon}$ is the dimensionless isentropic flow rate function, $y_{*}$ in Equation (8) is the width of region III in its "critical section" $O_{*} C_{*}$, and $M_{3}$ is the averaged flow Mach number after the curved shock $3\left(M_{3}=M_{3 T}\right.$ at first approximation). The static pressure $p$ at both sides of the slipstream and other flow parameters below it can be determined by isentropic formulas, for example:

$$
p / p_{T}=\pi\left(M_{3 \tau}\right) / \pi\left(M_{3}\right)=\pi\left(M_{2 \tau}\right) / \pi\left(M_{2 T}\right)
$$

Here, $\pi(M)=\left(1+\frac{\gamma-1}{2} M^{2}\right)^{-\gamma /(\gamma-1)}$ is the isentropic function of pressure, and $M_{2 \tau}$ is the flow Mach number in any point just upside the upstream.

If Mach stem height $y_{T}$ is sufficiently large comparing with flow cross-section size at whole, and flow gradients across region III are not negligible, some methods of flow averaging can be applied. At the very first, Mach number $M_{0}$ after the normal Mach shock at point $O$ can be used in Equations (9) and (10) instead of the Mach number $M_{3 T}$ just after the triple point; some averaged (between $M_{0}$ and $M_{3 T}$ ) values can be used also. However, because $M_{3 T}$ is really the initial Mach number below the slipstream that is to be correctly used in conditions of pressure equality at both its sides, the approximation that $M_{3} \equiv M_{0}$ is very disputable. As a compromise, the initial static pressure at the left side of subsonic zone III can be estimated not as pressure just after the triple point but as an averaged integral pressure [43]:

$$
p_{3}=\int_{0}^{y_{T}} p_{3}^{l}(y) d y / y_{T} .
$$

Classic shock relations supplemented by the parabolic approximation of the Mach shock shape can determine the local pressure $p_{3}^{l}(y)$ in any point after the curved Mach stem in Equation (11).

The original method of averaging the flow in the subsonic pocket is proposed and applied in $[26,28]$. Based on the first-order approximation of the stream to be averaged, authors derived the following relation to estimate the initial averaged Mach number:

$$
M_{3}=\frac{2\left(\rho_{3 T} u_{3 T} \cos \omega_{3 T}+\rho_{O} u_{O}\right)}{\left(\rho_{3 T}+\rho_{O}\right)\left(a_{3 T}+a_{O}\right)} .
$$


Here, $\rho_{3 T}, u_{3 T}$, and $a_{3 T}$ are the gas density, flow velocity, and sound speed in zone III just after the triple point; $\rho_{O}, u_{O}$, and $a_{O}$ are the corresponding parameters after the normal shock; $\omega_{3 T}$ is the slope angle of the Mach stem in the triple point.

Except Equations (11) and (12), some classical methods of flow averaging are also contained in [50]. We do not discuss the inevitable slipstream smearing and possible formation of Kelvin-Helmholtz instability along the contact discontinuity in this study [48,51], but it can be of sufficient interest later.

\subsection{Approximation of Flow Downstream the Reflected Shock}

Azevedo and Liu [43] proposed that it is the heading acoustical characteristic BC of the second family that falls onto the slipstream $\tau$ in the critical section of region III (i.e., that $C \equiv C_{*}$ in Figure $1 \mathrm{a}, \mathrm{b}$ ). All angles of inclination in the triangle BTC are easily calculable then:

- $\quad$ the angle of inclination of the slipstream $\tau$ to the horizontal plane is permanently equal to $\theta_{3 T}=\theta_{1}+\theta_{2 T}(5)$;

- $\quad$ the inclination angle of the reflected shock $T B$ is equal to $\omega_{2 T}-\theta_{1}$;

- $\quad$ the angle of inclination of the first incident characteristic $B C$ can be taken as $\beta_{2 B}-$ $\mu\left(M_{2 B}\right) \approx \theta_{3 T}-\mu\left(M_{3 T}\right)$ (in point $\left.B\right)$, or $\theta_{3 T}-\mu\left(M_{2 C *}\right)$, or any value averaged between above-mentioned ones. Here, $\mu(M)=\arcsin (1 / M)$ is the Mach angle, and $M_{2 C_{*}}$ is the flow Mach number that should be in the critical point $C_{*}$ above the slipstream $\tau$. According to static pressure equality on the slipstream, that Mach number can be calculated from the relation $\pi\left(M_{2 C_{*}}\right) / \pi\left(M_{2 T}\right)=\pi\left(M_{3 C_{*}}=1\right) / \pi\left(M_{3 T}\right)$.

Compared to the experimental results in $[12,13]$, large (up to $50 \%-100 \%$ ) errors in estimations provided by [43] are revealed. The primary reasons of those inadmissible mistakes are the following:

- $\quad$ the contact discontinuity $\tau$ is really the curvilinear one. Its slope angle is variable, and it is rather small initially (at the triple point). Therefore, an error in the slipstream slope angle estimation influences the estimation of length and width of the subsonic pocket crucially. Let us imagine, for example, a variation of the slipstream slope angle $\beta_{\tau}$ from $\beta_{3 T}=-2^{\circ}$ to $\beta_{\tau}=-10^{\circ}$ in point $C$. If we consider it constant, we mistake the length and width of the subsonic pocket several times. Therefore, neglecting slope angle variation leads to very large underestimation of the Mach stem size;

- $\quad$ the turn of the slipstream $\tau$ influenced by the incident expansion wave IV or V occurs not instantaneously but along the finite sector $C C_{*}$. As we neglect the length of the sector $C C_{*}$, it also leads to sufficient discrepancies.

The approximation [44] of flow in region II by Grib-Ryabinin method [52] based on the Lagrangian tangent transformation complicated the mathematical model sufficiently, but difference between results of $[43,44]$ is only $2-4 \%$ as it is demonstrated in [51], so the errors of Mach stem size estimation are basically preserved.

It is firstly proposed in $[45,53]$ and applied afterwards in $[25,28,54]$ to treat a flow in region II after reflected shock as a Prandtl-Meyer flow with straight acoustical characteristics of the first family. The proposed model is similar to the "shock-expansion method" [55-57], but it is applied not only for pressure estimation but also for restoring the whole geometry and corresponding features of all discontinuities. According to the Prandtl-Meyer invariant and condition of pressure equality across a slipstream, the following relations proven in [58] take place on the slipstream $\tau$ :

$$
\begin{gathered}
\frac{d y_{\tau}}{d x}=\tan \beta_{\tau}, \\
\frac{d \beta_{\tau}}{d x}=-\frac{\chi M_{3 \tau}^{2} \sqrt{M_{2 \tau}^{2}-1} \tan \beta_{\tau}}{M_{2 \tau}^{2}\left(M_{3 \tau}^{2}-1\right) y_{\tau}},
\end{gathered}
$$




$$
\begin{gathered}
\frac{d M_{2 \tau}}{d x}=\frac{\varphi\left(M_{2 \tau}\right) \tan \beta_{\tau}}{(1-\varepsilon) M_{2 \tau}\left(M_{3 \tau}^{2}-1\right) y_{\tau}}, \\
\frac{d M_{3 \tau}}{d x}=\frac{\varphi\left(M_{3 \tau}\right) M_{3 \tau}^{2} \tan \beta_{\tau}}{(1-\varepsilon)\left(M_{3 \tau}^{2}-1\right) y_{\tau}} .
\end{gathered}
$$

Here, $M_{2 \tau}$ and $M_{3 \tau}$ are the local Mach numbers above the slipstream and below it, correspondingly; $\varphi(M)=1+\varepsilon\left(M^{2}-1\right) ; y_{\tau}(x)$ is the equation for slipstream shape; $\beta_{\tau}(x)$ is the local angle of slipstream slope; $\chi=+1$ is the factor of the wave direction.

Equations (13)-(16) should be integrated until the slipstream interacts with the incident characteristic $B C$. As demonstrated in [53], we can reconstruct the shape of that curvilinear incident characteristic, solving the equations

$$
\begin{gathered}
\frac{d \beta_{w}}{d w}=\frac{2 \sqrt{M_{2 w}^{2}-1}}{M_{2 w}^{2}} \cdot N_{2}, \\
\frac{d M_{2 w}}{d w}=-\frac{2 \chi\left(1+\varepsilon\left(M_{2 w}^{2}-1\right)\right)}{(1-\varepsilon) M_{2 w}} \cdot N_{2}, \\
\frac{d \mu_{2 w}}{d w}=\frac{2 \chi\left(1+\varepsilon\left(M_{2 w}^{2}-1\right)\right)}{(1-\varepsilon) M_{2 w}^{2} \sqrt{M_{2 w}^{2}-1}}
\end{gathered}
$$

along its direction $w$ (see Figure 1a,b). Here, $\beta_{w}$ is the local flow angle; $M_{2 w}$ is the local Mach number; $\mu_{2 w}=\arcsin \left(1 / M_{2 w}\right)$ is the Mach angle; $N_{2}$ is the local streamline curvature. The equation

$$
\begin{gathered}
N_{2}=-\frac{\Psi(1-\varepsilon)\left(M_{2 w}^{2}-1\right) M_{3 w}^{2} \sin \left(\beta_{w}+\chi \mu_{2 w}\right) \sin \beta_{w}}{M_{2 w}^{2}\left[M_{2 w} M_{3 w}^{2} \sin \beta_{w} \cdot \Omega+\chi \Psi(1-\varepsilon) \sqrt{M_{2 w}^{2}-1}\left(M_{3 w}^{2}-1\right) \sin \left(\beta_{w}+\chi \mu_{2 w}\right)\right]}, \\
\Omega=y_{C_{1}}-y_{T} q\left(M_{3 T}\right) / q\left(M_{3 w}\right),
\end{gathered}
$$

determines the latter quantity in the voluntary point $C_{1}$. Here, $\Psi=+1$ because zone II is considered an expansion wave; $M_{3 w}$ depends on $M_{2 w}$ like $M_{3 \tau}$ depends on $M_{2 \tau}$, see Equations (9) and (10).

The results of the integration of Equations (13)-(16) witnessed $[53,58]$ that the contact discontinuity $\tau$ is always convex upwards, so its inclination to the horizontal plane increases along its length in the whole sector $T C$. The incident characteristic $B C$ is a weak discontinuity, not a strong one, so the instantaneous turn of the slipstream at point $C$ of its incidence is impossible.

\subsection{Reflected Shock Curvature}

As the expansion flow in region II influences the preceding reflected shock $T B$, its shape is curvilinear, and its strength $\left(J_{2}\right)$ and inclination angle to the incoming flow $\left(\omega_{2}\right)$ are variable. However, as it is proven, for example, in [8,57], the reflected disturbances in overtaking the shock-expansion interaction are very small (they are of third order compared to the shock wave and expansion one) and can be neglected. Consequently, the shape of the reflected shock can be restored in the following way: the flow direction at any point behind the shock should correspond to the flowfield in the following overtaking expansion wave II. The basics of this method and corresponding relations can be found in $[53,59]$.

Moreover, the strength of the influencing expansion flow at region BTH is rather small compared to other waves and shocks. As a result, a change in the slope angle of the reflected shock $T B$ is usually not more than $1^{\circ}$. Really, for example, at $M=5$ and $\sigma_{1}=40^{\circ}$ in the jet flow problem (dimensionless Mach stem size $y_{T} / h=0.390$ according to flow calculations by the method of characteristics for supersonic part of the flowfield $[47,58]$ ) reflected the shock inclination angle $\omega_{2 T}=40.857^{\circ}$ and inclination angle of the same shock at point $B$ of its intersection with the jet boundary $\omega_{2 B}=40,395^{\circ}$. At $M=5$ and $\sigma_{1}=40^{\circ}$, while the method $[49,60]$ leads to the value $y_{T} / h=0.589$, corresponding shock slope angles are equal to $\omega_{2 T}=47.492^{\circ}$ and $\omega_{2 B}=47.043^{\circ}$. For this reason, if we consider the reflected 
shock $T B$ as the straight one, with the slope angle permanently equal to its inclination angle in the triple point, as it is accepted in numerous studies [24-26,28,43,44,54,61,62], it does not lead to substantial error in Mach stem size.

If we do not intend to neglect the curvature of the reflected oblique shock, the method of its conjugation with the following overtaking expansion wave discussed in $[53,59]$ can be proposed. The shape of shock 2 can be found in the polar coordinate system $(r, \phi)$ connected with point $A$ from the solution of the following equations:

$$
\begin{gathered}
\frac{d r}{d \phi}=r \cdot \cot \left(\omega_{2}+\theta_{1}-\phi\right), \\
\frac{d \omega_{2}}{d \phi}=\frac{r}{\sin \left(\omega_{2}+\theta_{1}-\phi\right)} \cdot \Theta \cdot \frac{\partial \omega_{2}}{\partial \theta_{2}} .
\end{gathered}
$$

Here, $r$ is the distance from point $A$ to the current point of shock surface; $\omega_{2}$ is the current shock slope angle; $\Theta=M_{2} \cdot K_{S} \cdot \sin \left(\mu_{2}-\omega_{2}+\theta_{2}\right) ; \mu_{2}=\arcsin \left(1 / M_{2}\right) ; M_{2}$ is the current Mach number after the shock; $\theta_{2}$ is the current flow deflection angle; $K_{S}$ is the curvature of the streamline at the expansion wave after the shock:

$$
\begin{gathered}
K_{S}=-\frac{(1-\varepsilon)\left(M_{2}^{2}-1\right) M_{3}^{2} \sin \left(\beta+\mu_{2}\right) \sin \beta}{M_{2}^{2}\left[M_{2} M_{3}^{2} \sin \beta \cdot \Omega+(1-\varepsilon) \sqrt{M_{2}^{2}-1}\left(M_{3}^{2}-1\right) \sin \left(\beta+\mu_{2}\right)\right]} ; \\
\frac{\partial \omega_{2}}{\partial \beta_{2}}=\frac{M_{1}^{4}\left((1-\varepsilon) \cos ^{4} \omega_{2}-\left(1-2 \varepsilon^{2}\right) \cos ^{2} \omega_{2}-\varepsilon^{2}\right)-2 \varepsilon(1-\varepsilon) M_{1}^{2} \sin ^{2} \omega_{2}-(1-\varepsilon)^{2}}{(1-\varepsilon)\left[M_{1}^{4}\left((1+\varepsilon) \cos ^{4} \omega_{2}-(1+2 \varepsilon) \cos ^{2} \omega_{2}+\varepsilon\right)-M_{1}^{2}\left(2(1-\varepsilon) \cos ^{2} \omega_{2}-(1-2 \varepsilon)\right)-(1-\varepsilon)\right]},
\end{gathered}
$$

$\beta=\theta_{1}+\theta_{2}$ is the current flow direction angle after the shock; $q\left(M_{3}\right)$ is the flow rate function; $M_{3}$ depends on $M_{2}$ according to Equations (9) and (10); $\Omega=y-y_{T} q\left(M_{3 T}\right) / q\left(M_{3}\right)$; and $M_{3 T}$ is the flow Mach number after the Mach stem in the vicinity of the triple point.

Equations (18) and (19) are to be integrated from the value $\varphi_{T}=-\omega_{1}$ at the triple point $T$ (other initial conditions also correspond to the triple point) to the negative value $\varphi_{B}=\theta_{1}$ at point $B$.

The results achieved due to Equations (18) and (19) insufficiently (usually on $0.01-0.02^{\circ}$ for value of the angle $\omega_{2 B}$ ) differ from the results of calculations by the method of characteristics. This method allows determining not only coordinates of the point $B$ but also all flow parameters (for example, flow angle $\beta_{2 B}$ and Mach number $M_{2 B}$ ) after the shock at that point.

\subsection{Interaction of the Reflected Shock with the Rear Expansion Fan}

At least two ways exist to construct the shape of the reflected shock at the region of its interaction with the expansion fan IV of the opposite direction (see Figures 1a and 2, which cover this type of interaction specifically). The first of them was elaborated in $[63,64]$ and applied later in $[61,62]$ and further studies. It supposes the equality of static pressure and the co-direction of flow velocity vectors after the resulting shock $B_{1} B_{2}$ and refracted expansion wave $V$ that falls afterwards onto the slipstream $\tau$. The second one $[64,65]$ supposes that the resulting shock $B_{1} B_{2}$ after point $B_{2}$ is a straight one (i.e., it has zero geometrical curvature). Both methods lead to a second-order ordinary differential equation for the shape of the interacting shock $B B_{1}$. They also allow determining the flow parameters in the expansion wave $\mathrm{V}$.

The implementation of the first method $[63,64]$ looks as it follows. The so-called shear layer, bounded by weak tangential discontinuities $\tau_{1}$ and $\tau_{2}$, divides the flows that came through: centered expansion wave IV and resulting shock $8\left(B_{1} B_{2}\right)$, at one side; reflected shock $2(T B)$ and refracted wave V that are also considered like the Prandtl-Meyer flow at the other side of the shear layer. The conditions of pressure equality and flow co-direction downstream the resulting shocks and waves can be written in form:

$$
J_{2 B} J_{5}=J_{4} J_{8},
$$




$$
\theta_{2 B}+\theta_{5}=\theta_{4}+\theta_{8}
$$

At the oblique shocks considered in Equations (19) and (20),

$$
\begin{aligned}
\tan \left|\theta_{2 B}\right| & =\sqrt{\frac{(1+\varepsilon) M_{1}^{2}-J_{2 B}-\varepsilon}{J_{2 B}+\varepsilon}} \frac{(1-\varepsilon)\left(J_{2 B}-1\right)}{(1+\varepsilon) M_{1}^{2}-(1-\varepsilon)\left(J_{2 B}-1\right)}, \\
\tan \left|\theta_{8}\right| & =\sqrt{\frac{(1+\varepsilon) M_{4}^{2}-J_{8}-\varepsilon}{J_{8}+\varepsilon}} \frac{(1-\varepsilon)\left(J_{8}-1\right)}{(1+\varepsilon) M_{4}^{2}-(1-\varepsilon)\left(J_{8}-1\right)},
\end{aligned}
$$

In Prandtl-Meyer flows IV and V,

$$
\begin{aligned}
\theta_{4}=v\left(M_{4}\right)-v\left(M_{1}\right), \theta_{5} & =v\left(M_{2}\right)-v\left(M_{2 B}\right), J_{4}=\pi\left(M_{4}\right) / \pi\left(M_{1}\right), \\
J_{5} & =\pi\left(M_{2}\right) / \pi\left(M_{2 B}\right) .
\end{aligned}
$$

In Equations (20)-(24), $M_{4}$ and $M_{5}$ are flow Mach numbers after the waves IV and V; $J_{8}$ and $\theta_{8}$ are the strength of the resulting shock 8 in point $B_{1}$ and corresponding flow deflection angle. As the result of the solutions of Equations (20)-(24), we determine the strength $J_{8}$ of the resulting shock and its slope angle, as well as the strength $J_{5}$ of the refracted wave $\mathrm{V}$, the Mach number $M_{5}$ after it, and the slope angle of its rear straight acoustic characteristic because it is inclined at Mach angle to flow velocity vector determined due to Equation (21).

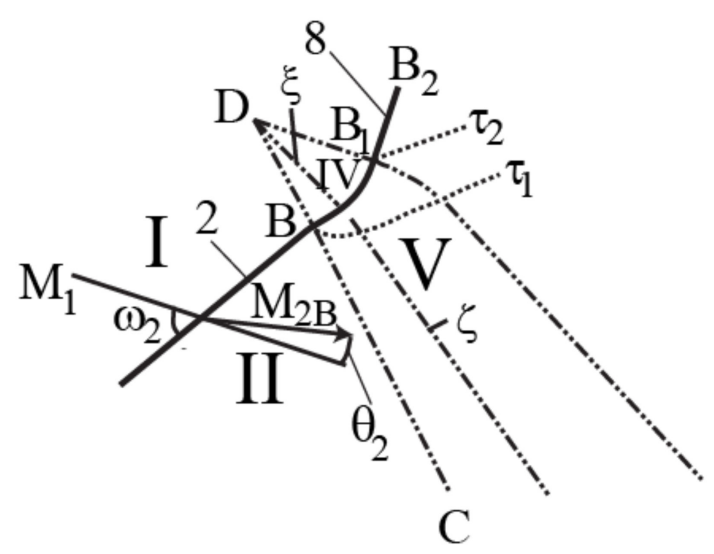

Figure 2. Oblique shock interaction with an expansion fan (fragment of Figure 1a with additional symbols). Here, $\mathrm{I}$ is the flow region downstream of the incident shock $\left(M_{1}\right.$ is the flow Mach number in that zone); II is the flow region downstream of the reflected shock 2; IV is the rear expansion fan ( $D$ is its center; $\xi$ is its voluntary straight second-family characteristic); $V$ is the refracted expansion fan ( $\zeta$ is its voluntary second-family characteristic); $B B_{1}$ is the curvilinear shock under influence of wave IV; $8\left(B_{1} B_{2}\right)$ is the resulting shock after that influence; $\tau_{1}$ and $\tau_{2}$ are weak tangential discontinuities that border a shear layer between them; $\omega_{2}$ and $\theta_{2}$ are, correspondingly, the slope angle and flow deflection angle on the shock $2 ; M_{2 B}$ is the flow Mach number in point $B$ immediately after that shock.

Without any additional stipulations, we can consider shock interaction with its part instead of the whole expansion wave of the opposite direction until the voluntary straight characteristic $\xi$ (see Figure 2). This characteristic with the local Mach number $M_{\xi}$ in inclined at angle $\theta_{1}+v\left(M_{\xi}\right)-v\left(M_{1}\right)-\mu\left(M_{\xi}\right)$ to the horizontal axis.

Solving the system which is completely analogous to Equations (20)-(24), we can obtain: flow Mach numbers an both sides of the interacting shock $B B_{1}$ at its voluntary point; its inclination angle, which leads to equation for $y^{\prime}{ }_{B B_{1}}(x)$ determining shock shape; the inclination angle of the voluntary characteristic $\varsigma$ of the refracted wave V (see Figure 2); the flow direction and Mach number along that characteristic. As it is demonstrated in [53,65], the obtained solutions are almost indiscernibly close to the exact one. 
The second method $[64,65]$ is based on the assumption that the oblique reflected shock is almost straight (i.e., it has zero curvature) just before point $B$ (shock 2 ) and just after point $B_{1}$ (shock 8$)$. Differential conditions of dynamic compatibility $[17,65,66]$ together with the precise decision of oblique shock-weak discontinuity interaction problem [67], in this case, allow the precise estimation of the geometrical curvature of the shock $B B_{1}$ in its voluntary point. It leads to second-order ordinary differential equation, which determines shock shape, strength, Mach numbers, and other variable flow parameters at both its sides with, as shown in $[53,65]$, almost the same accuracy or a little better.

\subsection{Expansion Wave Incidence and Reflection from the Slipstream}

Turn of the slipstream $\tau$ to the horizontal direction in point $C_{*}$ (see Figure $1 \mathrm{a}, \mathrm{b}$ ) under the influence of expansion wave IV (Figure 1b) or refracted expansion wave V (Figure 1a) cannot be described analytically with absolute accuracy. Authors of numerous approximate analytical models usually substitute the analytical research to numerical analysis, applying the numerical method of characteristics worked out for isentropic [24-26,28,61,62] and non-isentropic $[49,60]$ flows. On the other hand, we cannot neglect the finite length of the sector $C C_{*}$ because it leads to sufficient errors in Mach stem size as in $[43,44]$. Therefore, the interaction of the incident expansion wave with the slipstream should be studied additionally and obligatorily.

The analytical method to research the expansion wave reflection from the slipstream is described and discussed in $[45,53,59]$. Limiting ourselves to reflection of the centered wave and keeping the nomenclature of the study [60], as is shown in Figure 3, let us discuss this method, which can be generalized to non-centered wave reflection as it occurs in flow between two wedges.

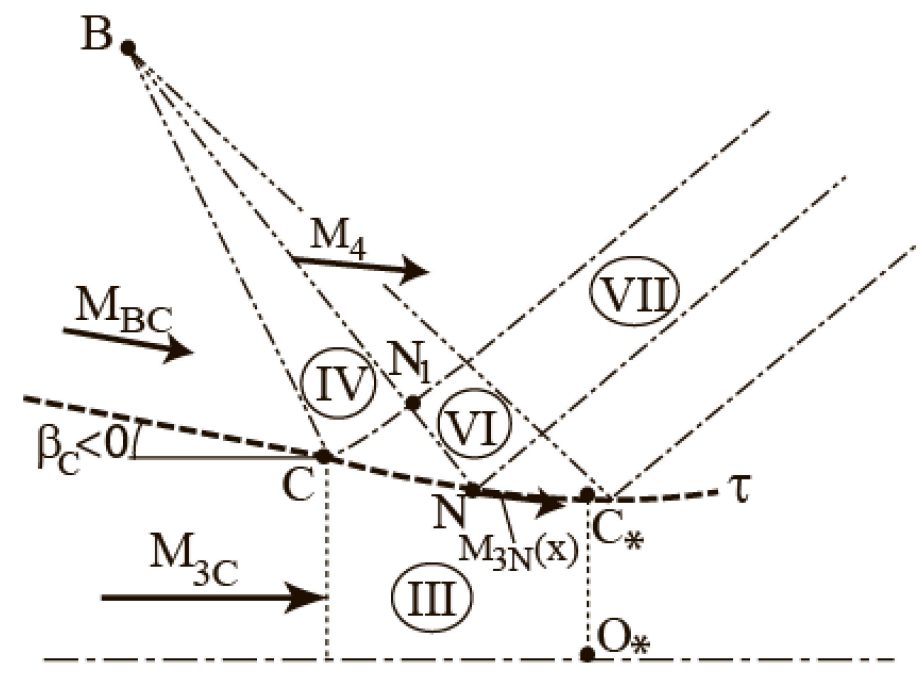

Figure 3. The incidence of expansion wave IV onto the border of the quasi-one-dimensional flow region III (fragment of Figure $1 \mathrm{~b}$ with additional symbols). Here, $\tau$ is the slipstream $\left(\beta_{C}\right.$ is its slope angle in point $C)$; III is the "virtual nozzle" $\left(C_{*} O_{*}\right.$ is its "critical section"; $M_{3 C}$ is the flow Mach number just below point $C ; M_{3 N}$ is the flow Mach number in the voluntary point $N$ just below the slipstream); IV is the incident expansion wave ( $B C$ is its heading characteristic; $M_{B C}$ is the Mach number on the characteristic $B C ; M_{4}$ is the flow Mach number on voluntary incident characteristic $B N_{1}$ ); VI is the region of interaction; VII is the reflected wave.

Calculations of the supersonic part of the flow reveal that the flow non-uniformities (the term accepted in $[17,65])$ after the reflected shock are small compared to the intensive variation of flow parameters in region IV (see Figure 1). For example, the sector TC of slow deflection of the stream at tangential discontinuity is several times longer than the sector $C_{*}$ of its opposite turn. Therefore, we can consider the flow parameters at the characteristic $B C$ as averaged and uniform. The characteristic line $B C$ itself can be treated 
as a straight one, and flow in region IV can be studied as a centered Prandtl-Meyer wave with straight characteristics of the second family.

The border characteristic $B C$ is also a weak discontinuity: streamlines suffer a finite break on curvature when crossing that line. In particular, the curvature of the slipstream $\tau$ changes abruptly and, as a rule, becomes positive in point $C$. Therefore, the tangential discontinuity $\tau$ abruptly becomes convex downwards.

The averaged flow Mach number $M_{B C}$ at the characteristic $B C$ is determined as follows:

$$
M_{B C}=1 / \sin \left(\beta_{C}+\arctan \Delta_{x y}+n \pi\right) .
$$

Here, $n=0$ at $x_{C}>x_{B} ; n=1$ elsewhere; $\Delta_{x y}=\left(y_{C}-y_{B}\right) /\left(x_{C}-x_{B}\right)$; and $\beta_{C}$ is the flow direction angle in point $C$.

The following relation is correct for the voluntary straight characteristic $B N_{1}$ inside expansion fan IV:

$$
\left(y_{N_{1}}-y_{B}\right) /\left(x_{N_{1}}-x_{B}\right)=\tan \left(\beta_{D}+v\left(M_{4}\right)-v\left(M_{B C}\right)-\mu\left(M_{4}\right)\right) .
$$
function.

Here, $v(M)=1 / \sqrt{\varepsilon} \arctan \sqrt{\varepsilon\left(M^{2}-1\right)}-\arctan \sqrt{M^{2}-1}$ is the Prandtl-Meyer

Equation (21) determines the flow Mach number $M_{4}$ at this voluntary incident characteristic. Numerical results of [53] allow recognizing the characteristic line $B N$ straight not only in region IV but also at sector $N_{1} N$ inside region VI. Connecting the flow deflection angle in the incident Prandtl-Meyer wave (IV) and in the reflected one (VII), it is easy to derive the following equation for the shape of the sector $D C$ of the tangential discontinuity $\tau$ :

$$
y_{\tau}^{\prime}\left(x_{\tau}\right)=\tan \left(\beta_{C}+2 v\left(M_{4}\right)-v\left(M_{B C}\right)-v\left(M_{N}\right)\right),
$$

So that, at the same time, the equation analogous to Equation (21)

$$
\left(y_{\tau}-y_{B}\right) /\left(x_{\tau}-x_{B}\right)=\tan \left(\beta_{C}+v\left(M_{4}\right)-v\left(M_{B C}\right)-\mu\left(M_{4}\right)\right)
$$

determines the flow Mach number $M_{4}$ in the incident wave. The Mach number $M_{N}$ at the upper side of the slipstream is to be determined from the condition of equality of the static pressures similar to Equation (10):

$$
\pi\left(M_{N}\right) / \pi\left(M_{B C}\right)=\pi\left(M_{3 N}\right) / \pi\left(M_{3 C}\right),
$$

Additionally, the Mach number $M_{3 N}$ at the lower side of the slipstream is to be determined by the mass conservation law in the following form:

$$
y_{N} / y_{C}=q\left(M_{3 N}\right) / q\left(M_{3 C}\right) .
$$

Equation (22), in addition to Equations (20), (21) and (23)-(25), allows constructing the slipstream after point $C$ until the flow below it would not reach the critical velocity value (i.e., until $M_{3 N}=1$ ).

Applying the decision of oblique shock interaction with the expansion fan of the opposite direction reached in $[63,64]$, it is easy to generalize Equations (20)-(25) for the slipstream under the influence of the refracted non-centered wave.

\subsection{General Algorithm}

The equations discussed above allow calculating the shock-wave structure of the whole studied flow, if only the governing conditions (values of the undisturbed flow Mach number $M$, the slope angle $\sigma_{1}$ of the incident shock, and the ratio $\gamma$ of the gas specific heats) are given. One unknown quantity (Mach stem height $y_{T}$ ) is to be appointed iteratively. The 
following final algorithm was proposed for accurate estimation of Mach stem height in gas jet flows $[45,53,59]$ and flows in restricting channels [53]:

1. Values of $M, \omega_{1}$, and $\gamma$ are given. The problem of Mach reflection of the shock 1 is decided using the triple-shock theory (1-8). In this way, the strengths $J_{2}$ and $J_{3}$ of the reflected shock and the Mach one in the vicinity of the triple point, as well as Mach numbers $M_{2 T}$ and $M_{3 T}$ downstream of those shocks, and the initial inclination angle $\beta_{3 T}$ of the slipstream $\tau$ are to be determined.

2. Some initial estimation value of $y_{T}$ (Mach stem height at the first iteration) is to be appointed (pre-estimated).

3. The shape of the reflected shock at sector $T B$ can be determined by Equations (18) and (19). Those equations are to be integrated until the overexpanded jet boundary or the point of shock intersection with the first characteristic of the tail expansion fan (i.e., till the point $B$ ). The flow Mach number $M_{2 B}$ and flow direction angle $\beta_{2 B}$ after the shock in point $B$ are to be calculated.

4. Equations (13)-(16), which determine the shape of the slipstream $\tau$ at its sector TC (as well as flow features on both its sides), are to be integrated. Equation (17), which determine the shape of the first incident characteristic $B C$ (and flow features along it), are to be decided simultaneously until point $C$ of the intersection of the incident characteristic with the slipstream.

5. Only in the problem of the flow between two wedges, we need to decide the problem of the oblique shock interaction with the expansion fan. Applying the methods elaborated by Li and Ben-Dor [63] or by Meshkov and Omelchenko [64], we restore the shape of the interacting shock $B B_{1}$ and flow parameters in the refracted expansion wave V.

6. Flow characteristics at the first border characteristic $B C$ of the incident expansion fan are to be averaged. Equations (20)-(25) determine the shape of the slipstream $\tau$ at its sector $C C_{*}$. We solve this equation until one of two following conditions would not be fulfilled: full horizontal turn of the slipstream $\tau\left(\beta_{\tau}=0\right)$ or increase in the flow velocity of its lower side to the critical value $\left(M_{3 \tau}=1\right)$. In the first case, the current Mach stem height $y_{T}$ is considered as too large; at second case, it is considered as too small.

7. With the above-mentioned conclusion about the value of $y_{T}$, we correct the proposing Mach stem height and return to point 3. The result of the last iteration (when achieving enough accuracy) is considered the final one.

Therefore, the problem of Mach stem height calculation (and corresponding analysis of the whole flowfield) is reduced to its estimation in the boundary value problem for several ordinary differential equations.

Our calculation demonstrated that there are no numerical instabilities at reasonably given flow parameters, at least, in overexpanded supersonic jet flow with a Mach number $M>2$ when the incident shock strength satisfies the inequality in Equation (8). The selection of the Mach stem size with ordinary PC and chosen widespread software (MATLAB 2017) takes about one minute, while it takes several hours to select the Mach stem size applying the second-order method of characteristics at every step. The direct CFD calculation with well-known hydrocodes (for example, ANSYS Fluent applied in [32] to similar problem) takes about two hours at the same PC, but it does not allow estimating the Mach stem size and shape of other gasodynamic discontinuities due to insufficient shock resolution of widely used shock-capturing methods.

\section{Results}

The results of the calculation of the Mach stem size $y_{T}$ in overexpanded jet flow (divided on nozzle half-width $h$ ) are presented in Table 1 (see also Figure 4) for flow Mach number $M=5$ and various angles $\omega_{1}$ of the incident shock inclination [59]. The results achieved by the numerical method of characteristics for all supersonic flowfields [49] are presented in the last string of Table 1 ; they differ approximately on $0.5 \%$. The authors also 
realized the second-order method of characteristics for the supersonic part of the flow. The results of comparison between the authors' numerical and analytical data are presented in Figure 4 , and the difference between them also does not exceed $1 \%$. Consequently, proposing the approximate analytical method has very large accuracy. For example, at the similar problem of Mach reflection in planar narrowing channel imitating the air inlet $[43,44]$, the results of the actual approximate analytical solutions differs from numerical and experimental data by $30 \%-40 \%$ and even by $90 \%$. Flow calculations at Mach numbers $M=3$ and $M=4$ demonstrated even larger accuracy than Table 1 presents.

Table 1. Comparison of analytical method with numerical results.

\begin{tabular}{cccccccccc}
\hline $\boldsymbol{\omega}_{\mathbf{1}}{ }^{\circ}$ & $\mathbf{3 1}$ & $\mathbf{3 5}$ & $\mathbf{3 9}$ & $\mathbf{4 3}$ & $\mathbf{4 7}$ & $\mathbf{5 1}$ & $\mathbf{5 5}$ & $\mathbf{5 9}$ \\
\hline$y_{T} / h$, proposed method & 0.046 & 0.243 & 0.363 & 0.455 & 0.532 & 0.602 & 0.673 & 0.753 \\
\hline$y_{T} / h$, method of characteristics & 0.046 & 0.245 & 0.364 & 0.457 & 0.536 & 0.607 & 0.677 & 0.756 \\
\hline
\end{tabular}

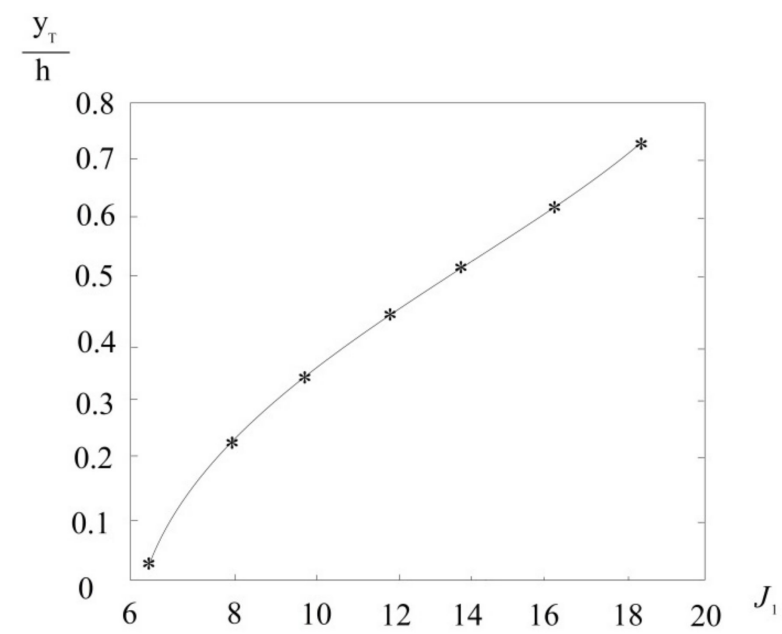

Figure 4. Values of dimensionless Mach stem height $\left(y_{T} / h\right)$ determined analytically (solid line) and numerically (stars) vs. the strength of the incident shock $\left(J_{1}\right)$.

As Figure 4 demonstrates, the Mach stem height is a continuous function of the angle $\omega_{1}$, starting from the value $y_{T}=0$ at $\omega_{1}=30.796^{\circ}$, which corresponds to the von Neumann criterion of shock reflection transition.

We should remark that, at large values of the incident shock $\left(\omega_{1}=47^{\circ}\right.$ and larger), expansion fan IV does not completely turn the slipstream to the horizontal direction with the contemporary transition of flow in region III through the critical velocity. The presented solutions are based on the artificial prolongation of the expansion fan. We suppose that gas flow in the "virtual nozzle" can remain subsonic at very deep overexpansion (flow separation inside the nozzle is not studied here).

The values of the dimensionless Mach stem height in the narrowing channel are given in Figure 5 depending on the incident shock inclination angle $\left(\omega_{1}\right)$ at flow Mach number $M=3.98$. The results shown in Figure 5 lie within measurement errors of the experimental data of [12], whilst the results of the analytical model [28] differ from them by $20-25 \%$, and discrepancies of approximate models $[43,44]$ are even more. 


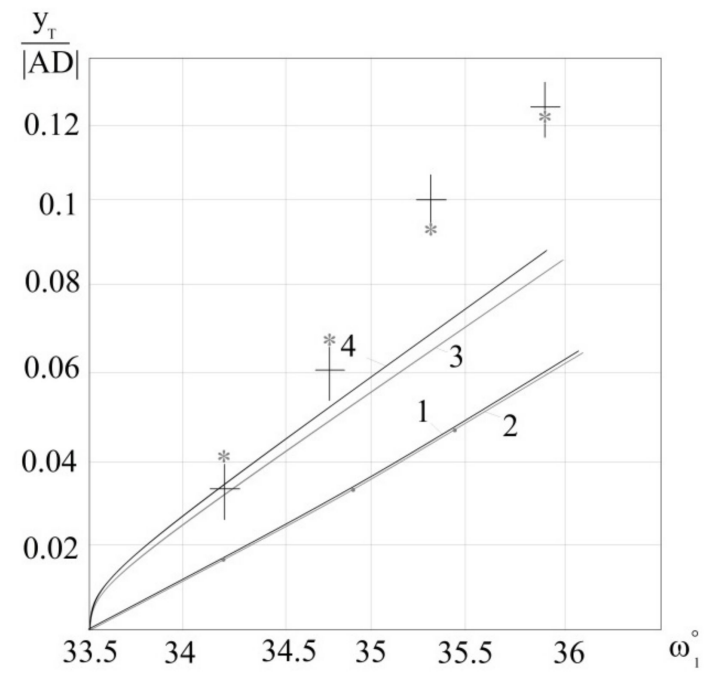

Figure 5. The values of the dimensionless Mach stem height $\left(y_{T} /|A D|\right)$ at gas flow in the narrowing channel vs. incident shock inclination angle $\left(\omega_{1}\right)$. The flow Mach number $M=3.98$; large crosses are the experimental data of [12]; curve 1 corresponds to the engineering approach of [43]; circles and approximating curve 2 correspond to the method of [44]; curve 3 are the results of method [28]; curve 4 are data of [53].

\section{Conclusions}

Based on the results of the decision of the separate problems outnumbered earlier, including the triple-point solution, the conjugation of Prandtl-Meyer wave with preceding overtaking shocks and with quasi-one-dimensional flow, and the interaction of the incident expansion wave with the slipstream, we worked out a new complex analytical model for supersonic flow with Mach reflection. The results obtained for supersonic overexpanded jet flow demonstrated its high accuracy, especially in the determination of the Mach stem size value.

The next necessary step for adaptation of this analytical model for real flows in ramjets and detonation engines is to consider the impulse heat influx at the Mach stem, as well as real gas effects at large Mach numbers. It can also be useful to apply the amendments made by Russian, Chinese, and Indian authors for asymmetrical and axisymmetric flows with irregular shock reflection.

Author Contributions: Conceptualization, M.V.C.; methodology, M.V.C.; software, M.V.C.; validation, A.S.K. and K.E.S.; formal analysis, M.V.C.; resources, A.S.K.; writing-original draft preparation, K.E.S.; writing-review and editing, M.V.C.; supervision, M.V.C.; funding acquisition, M.V.C. All authors have read and agreed to the published version of the manuscript.

Funding: This study was financially supported by the Ministry of Science and Higher Education of the Russian Federation during implementation of the project "Creating a leading scientific and technical reserve in the development of advanced technologies for small gas turbine, rocket and combined engines of ultra-light launch vehicles, small spacecraft and unmanned aerial vehicles that provide priority positions for Russian companies in emerging global markets of the future", No. FZWF-2020-0015.

Conflicts of Interest: The authors declare no conflict of interest.

\section{References}

1. Mach, E. Über den verlauf von Funkenwellen in der Ebene und im Raume. Sitzungsbr. Akad. Wiss. Wien. 1878, 78, 819-838. (In German)

2. Reichenbach, H. Contribution of Ernst Mach to fluid mechanics. Annu. Rev. Fluid Mech. 1983, 15, 1-28. [CrossRef]

3. Pack, D.C. The reflection and diffraction of shock waves. J. Fluid Mech. 1964, 18, 549-576. [CrossRef]

4. Griffith, W.C. Shock waves. J. Fluid Mech. 1981, 106, 81-101. [CrossRef] 
5. Hornung, H.G. Regular and Mach reflection of shock waves. Annu. Rev. Fluid Mech. 1986, 18, 33-58. [CrossRef]

6. Ben-Dor, G. Shock Wave Reflection Phenomena; Springer: Berlin/Heidelberg, Germany; New York, NY, USA, 2007; 342p.

7. Von Neumann, J. Oblique Reflection of Shock Waves; Collecttion Works; Pergamon Press: London, UK, 1963; Volume 6, pp. 238-299.

8. Courant, R.; Friedrichs, K.O. Supersonic Flow and Shock Waves; Springer: New York, NY, USA, 1976; 464p.

9. Landau, L.D.; Lifshitz, E.M. Course of Theoretical Physics; Fluid Mechanics; Pergamon Press: Oxford, UK, 1987; Volume 6, 552p.

10. Zel'dovich, Y.B.; Raizer, Y.P. Physics of Shock Waves and High-Temperature Hydrodynamic Phenomena; Academic Press: New York, NY, USA, 1966; Volume I, 464p.

11. Melnikov, D.A. Reflections of shock waves from the axis of symmetry. J. USSR Acad. Science. Mech. Mech. Eng. 1962, 3, 24-30. (In Russian)

12. Hornung, H.G.; Oertel, H.; Sandeman, R.J. Transition to Mach reflexion of shock waves in steady and pseudosteady flow with and without relaxation. J. Fluid Mech. 1979, 90, 541-560. [CrossRef]

13. Hornung, H.G.; Robinson, M.I. Transition from regular to Mach reflection of shock waves. Part 2: The steady-flow criterion. J. Fluid Mech. 1982, 123, 155-164. [CrossRef]

14. Chpoun, A.; Ben-Dor, G. Numerical confirmation of the hysteresis phenomenon in the regular to Mach reflection transition in steady flows. Shock Waves 1995, 5, 199-204. [CrossRef]

15. Ivanov, M.S.; Gimelshein, S.F.; Beylich, A.E. Hysteresis effect in stationary reflection of shock waves. Phys. Fluids 1995, 7, 685-687. [CrossRef]

16. Shimshi, E.; Ben-Dor, G.; Levy, A. Viscous simulation of shock-reflection hysteresis in overexpanded planar nozzles. J. Fluid Mech. 2009, 635, 189-206. [CrossRef]

17. Adrianov, A.L.; Starykh, A.L.; Uskov, V.N. Interference of Stationary Gasodynamic Discontinuities; Nauka: Novosibirsk, Russia, 1995; 180p. (In Russian)

18. Uskov, V.N.; Chernyshov, M.V. Special and extreme triple shock-wave configurations. J. Appl. Mech. Tech. Phys. 2006, 47, 492-504. [CrossRef]

19. Chernyshov, M.V. Extreme Triple Configurations with Negative Slope Angle of the Reflected Shock. Russ. Aeronaut. 2019, 62, 259-266. [CrossRef]

20. Shoev, G.V.; Ivanov, M.S. Numerical study of shock wave interaction in steady flows of a viscous heat-conducting gas with a low ratio of specific heats. Thermophys. Aeromech. 2016, 23, 343-364. [CrossRef]

21. Gvozdeva, L.G.; Silnikov, M.V.; Gavrenkov, S.A. Triple shock configurations with negative angle of reflection. Acta Astronaut. 2015, 116, 36-42. [CrossRef]

22. Gavrenkov, S.A.; Gvozdeva, L.G. Numerical investigation of the onset of instability of triple shock configurations in steady supersonic gas flows. Tech. Phys. Lett. 2012, 38, 587-589. [CrossRef]

23. Chernyshov, M.V.; Kapralova, A.S.; Savelova, K.E. Ambiguity of solution for triple configurations of stationary shocks with negative reflection angle. Acta Astronaut. 2021, 179, 382-390. [CrossRef]

24. Tao, Y.; Liu, W.; Fan, X.; Xiong, B.; Yu, J.; Sun, M. A study of the asymmetric shock reflection configurations in steady flows. J. Fluid Mech. 2017, 825, 1-15. [CrossRef]

25. Lin, J.; Bai, C.-Y.; Wu, Z.-N. Study of asymmetrical shock wave reflection in steady supersonic flow. J. Fluid Mech. 2019, 864, 848-875. [CrossRef]

26. Roy, S.; Gopalapillai, R. An analytical model for asymmetric Mach reflection configuration in steady flows. J. Fluid Mech. 2019, 863, 242-268. [CrossRef]

27. Gounko, Y.P. Patterns of steady axisymmetric supersonic compression flows with a Mach disk. Shock Waves 2017, 27, 495-506. [CrossRef]

28. Choe, S.-G. A method for predicting Mach stem height in steady flows. Proc. Inst. Mech. Eng. Part G J. Aerosp. Eng. 2021. Available online: https: / / doi.org/10.1177/09544100211001518 (accessed on 27 March 2021). (in “OnlineFirst" now). [CrossRef]

29. Bulat, P.V.; Volkov, K.N. Detonation jet engine. Part II-Construction features. Int. J. Environ. Sci. Educ. 2016, 11, $5020-5033$.

30. Ivanov, M.S.; Kudrjavtsev, A.N.; Trotsjuk, A.V.; Fomin, V.M. Method of Organization of Detonation Combustion Chamber of Supersonic Ramjet. Engine. Patent RU 2285143 C2, 10 December 2004. (In Russian).

31. Chernyshov, M.V.; Murzina, K.E.; Matveev, S.A.; Yakovlev, V.V. Shock-wave structures of prospective combined ramjet engine. IOP Conf. Ser. Mater. Sci. Eng. 2019, 618, 012068. [CrossRef]

32. Savelova, K.E.; Alekseeva, M.M.; Matveev, S.A.; Chernyshov, M.V. Shock-wave structures of prospective combined jet engine. J. Phys. Conf. Ser. 2021, 1959, 012043. [CrossRef]

33. Prakash, A.; Parsons, N.; Wang, X.; Zhong, X. High-order shock-fitting methods for direct numerical simulation of hypersonic flow with chemical and thermal nonequilibrium. J. Comput. Phys. 2011, 230, 8474-8507. [CrossRef]

34. Pepe, R.; Bonfiglioli, A.; D'Angola, A.; Colonna, G.; Paciorri, R. An unstructured shock-fitting solver for hypersonic plasma flows in chemical non-equilibrium. Comput. Phys. Commun. 2015, 196, 179-193. [CrossRef]

35. Li, H.; Ben-Dor, G.; Grönig, H. Analytical Study of the Oblique Reflection of Detonation Waves. AIAA J. 1997, 35, 1712-1720. [CrossRef]

36. Medvedev, A.E. Reflection of an oblique shock wave in a reacting gas with a finite relaxation-zone length. J. Appl. Mech. Tech. Phys. 2001, 42, 211-218. [CrossRef] 
37. Godunov, S.K.; Zabrodin, A.V.; Ivanov, M.Y.; Kraiko, A.N.; Prokopov, G.P. Numerical Solution of Multidimensional Problems of Gas Dynamics; Nauka: Moscow, Russia, 1976; 400p. (In Russian)

38. Kozhemyakin, A.O.; Omel'chenko, A.V.; Uskov, V.N. Oblique interaction of supersonic flows. Fluid Dyn. 1999, 34, 551-557.

39. Chernyshov, M.V.; Tolpegin, O.A. Optimal regular reflection of oblique shocks. Acta Astronaut. 2019, 163, 225-231. [CrossRef]

40. Henderson, L.F. On the confluence of three shock waves in a perfect gas. Aeronaut. Q. 1964, 15, 181-197. [CrossRef]

41. Hekiri, H.; Emanuel, G. Shock wave triple point morphology. Shock Waves 2011, 21, 511-521. [CrossRef]

42. Hekiri, H.; Emanuel, G. Structure and morphology of a triple point. Phys. Fluids 2015, 27, 056102. [CrossRef]

43. Azevedo, D.J.; Liu, C.S. Engineering approach to the prediction of shock patterns in bounded high-speed flows. AIAA J. 1993, 31, 83-90. [CrossRef]

44. Medvedev, A.E.; Fomin, V.M. Approximate analytical calculation of the Mach configuration of steady shock waves in a plane constricting channel. J. Appl. Mech. Tech. Phys. 1998, 39, 369-374. [CrossRef]

45. Omel'chenko, A.V.; Uskov, V.N.; Chernyshev, M.V. An Approximate Analytical Model of Flow in the First Barrel of an Overexpanded Jet. Tech. Phys. Lett. 2003, 29, 243-245. [CrossRef]

46. Ben-Dor, G.; Takayama, K. The phenomena of shock wave reflection-A review of unsolved problems and future research needs. Shock Waves 1992, 2, 211-223. [CrossRef]

47. Chanetz, B.; Chernyshev, M.V.; Durand, A.; Ivanov, M.S.; Khotyanovsky, D.V.; Kudryavtsev, A.N.; Omelchenko, A.V.; Uskov, V.N. Analytical, Numerical, and Experimental Investigation of Shock Wave Reflection Transition Induced by Variation of Distance between Wedges, Proceedings of West East High Speed Flow Fields 2002 Conference, Marseille, France, 22-26 April 2002; CIMNE: Barcelona, Spain, 2003; pp. 274-281.

48. Hadjadj, A.; Kudryavtsev, A.N.; Ivanov, M.S. Numerical Investigation of Shock-Reflection Phenomena in Overexpanded Supersonic Jets. AIAA J. 2004, 42, 570-577. [CrossRef]

49. Avduevsky, V.S.; Ashratov, E.A.; Pirumov, U.G. Supersonic Non-Isobaric Gas Jets; Mashinostroenie: Moscow, Russia, 1985; 248p. (In Russian)

50. Sedov, L.I.; Cherny, G.G. On averaging of non-uniform gas streams in channels. Theor. Hydromech. 1954, 12, 27-45. (In Russian)

51. Yuan, T.; Xiaoqiang, F.; Yilong, Z. Flow visualization for the evolution of the slipstream in steady shock reflection. J. Vis. 2014, $18,21-24$.

52. Grib, A.A.; Ryabinin, A.G. Approximate integration of the equations of steady supersonic gas flow. Dokl. Akad. Nauk SSSR 1955, 100, 425-428. (in Russian).

53. Chernyshov, M.V. Interaction of Elements of Shock-Wave Systems between Themselves and with Various Surfaces. Ph.D. Thesis, Saint Petersburg State University, Saint Petersburg, Russia, 2002; 173p. (In Russian).

54. Thomas, T.Y. First approximation of pressure distribution on curved profiles at supersonic speeds. Proc. Natl. Acad. Sci. USA 1949, 35, 617-627. [CrossRef] [PubMed]

55. Meyer, R.E. On supersonic flow behind a curved shock. Q. Appl. Math. 1957, 14, 433-436. [CrossRef]

56. Cherny, G.G. Gas Flows with Large Supersonic Velocity; FIZMATGIZ: Moscow, Russia, 1959; 220p. (In Russian)

57. Bai, C.-Y.; Wu, Z.-N. Size and shape of shock waves and slipline for Mach reflection in steady flow. J. Fluid Mech. 2017, 818, 116-140. [CrossRef]

58. Silnikov, M.V.; Chernyshov, M.V. The interaction of Prandtl-Meyer wave and quasi-one-dimensional flow region. Acta Astronaut. 2015, 109, 248-253. [CrossRef]

59. Alekseeva, M.M.; Matveev, S.A.; Savelova, K.E.; Chernyshov, M.V. Interaction of Steady Gasodynamic Discontinuities and Waves on Supersonic Gas Jet Flows from Rocket Engines. AIP Conf. Proc. 2021, 2318, 060004.

60. Averenkova, G.I.; Ashratov, E.A.; Volkonskaya, T.G. Supersonic Jets of Ideal Gas; Part 2; Moscow State University: Moscow, Russia, 1971; 170p. (In Russian)

61. Li, H.; Ben-Dor, G. A parametric study of Mach reflection in steady flows. J. Fluid Mech. 1997, 341, 101-125. [CrossRef]

62. Schotz, M.; Levy, A.; Ben-Dor, G.; Igra, O. Analytical prediction of the wave configuration size in steady Mach reflection. Shock Waves 1997, 7, 363-372. [CrossRef]

63. Li, H.; Ben-Dor, G. Oblique Shock-Expansion Fan Interaction-Analytical Solution. AIAA J. 1996, 43, 418-421. [CrossRef]

64. Meshkov, V.R.; Omelchenko, A.V.; Uskov, V.N. The interaction of the stationary shock with the expansion wave of opposite direction. Vestn. St. Petersburg Univ. Math. 2002, 2, 99-106. (in Russian).

65. Silnikov, M.V.; Chernyshov, M.V.; Uskov, V.N. Analytical solutions for Prandtl-Meyer wave-oblique shock overtaking interaction. Acta Astronaut. 2014, 99, 175-183. [CrossRef]

66. Silnikov, M.V.; Chernyshov, M.V. Supersonic flow gradients at an overexpanded nozzle lip. Shock Waves 2018, $28,765-784$. [CrossRef]

67. Omel'chenko, A.V. Relation between derivatives across a strong discontinuity. Comput. Math. Math. Phys. 2002, $42,1200-1211$. 